\title{
Comparative studies in simple oddity learning: II. Children, adults and seniles'
}

PASCHAL N. STRONG, JR.

TEXAS TECHNOLOGICAL COLLEGE ${ }^{2}$

One hundred thirteen humans from the age of 3-0 to hospitalized senile patients were run on identical 1 trial, 2 position oddity problems used previously with cats, raccoons, monkeys, and chimpanzees. The youngest child to learn was 3 years, 4 months of age. Sixty-four percent of the 6 year olds learned while all 12 year olds and college students reached criterion performance within the second day. Some seniles learned the problem but showed atypical, insight like, learning curves.

Recently Strong \& Hedges (1966) pointed out the difficulty in comparing oddity learning in different species because of the difference in stimuli, presentation modes, and trials per problem used by various investigators. The present study is an extension of the author's previous works with animals.

Subjects

Table 1 indicates the number of Ss used in each age category. The pre-school children were obtained from three day care nurseries, two United Fund agencies, and the Texas Tech nursery. Ss from the former were Caucasian, Latin-American or Negro and came from lower socio-economic homes. The latter were children of faculty at Texas Tech. Of the 68 children in this group, 54 had IQ's determined by the Stanford Binet, form $L$.

The 12 year olds were obtained from a bible school class during the summer and were from middle class backgrounds.

The college group was comprised of Texas Tech male freshmen.

The senile group was obtained from Big Springs State Hospital in Texas. They were all 65 years or older and diagnosed as senile dementia and/or cerebral arteriosclerosis.

\section{Apparatus}

With the pre-school children, original learning was on one of two apparatuses, a modified WGTA similar to that used with chimpanzees in a previous study (Strong \& Hedges, 1966), and a Primate Automatic Testing Apparatus-Key (PATA-K) described by Strong (1965) and modified so as to be portable. This latter device presented two dimensional stimuli identical to the three dimensional WGTA stimuli. The same stimuli and problems used in the two previous studies cited above were used. Only the WGTA was used with the 12 year old and older Ss.

Procedure

On the first day Ss were simply told that they would play a game with the experimenter in the next room and that the experimenter would not be able to talk to the $\mathrm{S}$ or answer questions. E never spoke to $\mathrm{S}$ during the testing. Forty-eight trials were given each day of 2 position, one trial oddity problems as previously defined by Strong (1965). All pretraining procedures were identical to the previously cited studies. Children were rewarded with $M$ \& $M$ 's while adults were rewarded with candy, cigarettes or pennies according to their preference. Testing was discontinued if progress was not shown in 20 sessions.

\section{Results}

Table 1 summarizes the results of the study. Four modes of performance were noted.

1. Fast learners who attained criterion within the first two days, the minimum amount of time. The youngest S showing this had a CA of 4-7 and a MA of 5-4.

2. Slow learners who showed curves basically like those of infrahuman primates. The slowest human learner learned sooner (1248 trials) than our fastest infrahuman primate in past studies (1296 trials).

3. Non-learners who failed to learn the problem or show any significant progress in 20 sessions. Most of these Ss developed emotional behavior patterns after repeated failures.

4. A retrieval mode seen in seniles is indicated in

Table 1. Summary of Oddity Learning and Transfer in Humans

\begin{tabular}{lcccccrr} 
Age Group & $N$ & $\begin{array}{c}\text { Original } \\
\text { WGTA }\end{array}$ & $\begin{array}{c}\text { Apparatus } \\
\text { PATA-K }\end{array}$ & $\begin{array}{c}\text { Number Reaching } \\
\text { Criterion } \\
\text { WGTA }\end{array}$ & $\begin{array}{c}\bar{X} \text { Trials } \\
\text { PATA-K }\end{array}$ & $\begin{array}{c}\text { Standard } \\
\text { to Criterion* }\end{array}$ \\
\hline 3 years & 12 & 6 & 6 & 2 & 0 & 816.0 & -- \\
4 years & 18 & 12 & 6 & 6 & 1 & 397.7 & 410.0 \\
5 years & 27 & 18 & 9 & 9 & 4 & 372.9 & 395.0 \\
6 years & 11 & 6 & 5 & 2 & 5 & 150.9 & 97.6 \\
12 years & 11 & 11 & - & 11 & & 105.6 & 20.5 \\
College & 14 & 14 & & 14 & & 96.0 & 0.0 \\
Seniles & 20 & 20 & & 8 & 510.0 & 533.0 \\
\hline
\end{tabular}

- Includes 2 criterion sessions (96 trials) and only includes subjects solving problem. 


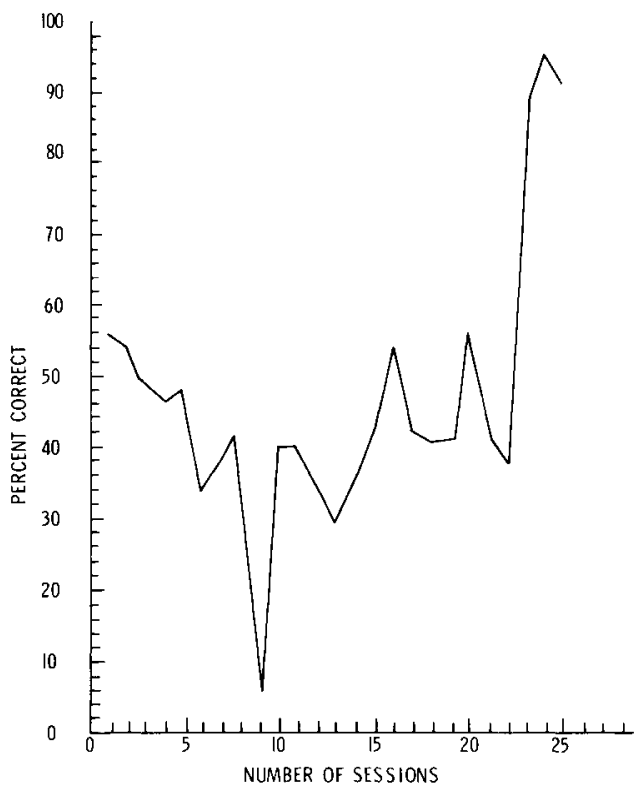

Fig. 1. Typical insight like leaming curve for senile learners.

Fig. 1. Performance shows several sessions at chance with a sudden grasping of the problem yielding an insight-like curve. Apparently these Ss are not learning oddity but merely retrieving a past concept and applying it to the appropriate situation.

For the three and four year old children it was apparent the WGTA apparatus leads to a significantly greater number Ss reaching criterion than the PATA-K. (P Fisher's exact test $=.03$. ) There was no significant difference between the two apparatuses for the five and six year olds. This is in contrast with Strong's (1965) previous findings for monkeys. Perhaps younger monkeys would show better performance with the WGTA three dimensional stimuli.

There was a suggestion that IQ did discriminate between the fast and slow learners ( $\bar{X}$ IQ fast learners = 115.4, $\overline{\mathrm{X}}$ IQ slow learners $=105.1$ ) but this missed the .05 level of significance $(t=1.79)$. However, the three best slow learners learned in three sessions and had IQ's of 125,124 and 132, respectively. Thus the relationship of IQ to rapid attainment of the oddity solution for pre-school children is probably a real one. There was no significant difference between the IQ's of slow learners and non-learners.

Figure 2 shows a summary of the comparative date to date. The choice of linear coordinates is, of course, arbitrary.

Discussion

Extension of oddity problems to humans indicates the young children tend to learn oddity in the same manner

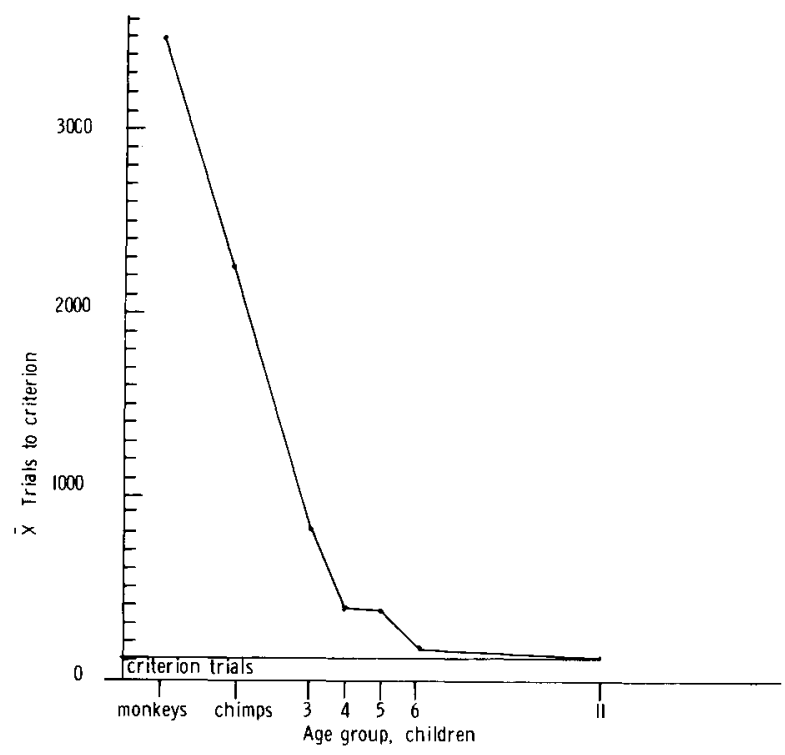

Fig. 2. Mean trials to criterion for various species.

as infrahuman primates. While they require fewer trials the distributions almost overlap between the fastest infrahuman primate and the slowest human and suggests differences are qualitative, not quantitative.

After a certain age or stage of development, between the age of five and seven probably, a new mode develops in which the first tendency is to choose the odd object and the solution is immediately apparent. Oddity, as a concept, is present and it merely must be reinforced once for it to become associated to the problem.

Of course, it would have been ideal if our non-learners could have been run for more sessions but resistance to testing and the need to maintain a good working relationship with cooperating agencies made discretion the better part of valor. When the child was willing, he was run for more sessions. One non-learner (CA 5-2, IQ 102) was run for 30 sessions (1440 trials) with no evidence of learning.

\section{References}

Strong, P. N. Learning and transfer of oddity as a function of apparatus and trials per problem. Psychon. Sci., 1965, 3, 19-20.

Strong, P. N., \& Hedges, M. Comparative studies in simple oddity learning I. Cats, raccoons, monkeys, and chimpanzees. Psychon. Sci., 1966, 5, 13-14.

\section{Notes}

1. This work was supported under Air Force Contract No. 29(600)2939. Grateful acknowledgment is made to the Comparative Psychology Laboratory personnel at Halloman AFB for their cooperation and encouragement.

2. Now at the University of South Florida. 